\title{
REFERENCE NUCLEAR DATA FOR SPACE APPLICATIONS
}

\author{
Sol Pearlstein \\ National Nuclear Data Center, Brookhaven National Laboratory, \\ Upton, NY 11973
}

\section{ABS TRACT}

The National Nuclear Data Center (NNDC) at Brookhaven Nationa? Laboratory is active in the development of high energy data bases for space applications. Validated data and methods of interaction analysis are needed to explain and predict radiation patterns. The NNDC uses methods consisting of nuclear systematics and nuclear model codes to provide neutron and proton induced reaction data from $1 \mathrm{MeV}$ to $1 \mathrm{GeV}$. The data can be placed in convenient form for use by radiation transport codes. In addition to cross-sections, nuclear structure and radioactive decay data are also stored in data bases. Data are distributed by the NNDC in a variety of ways including on-line access through computer networks or telephone lines.

Nuclear reaction, nuclear structure, and radioactive decay data are needed to explain the origin and composition of background radiation in space. Since it is not possible to measure everything of importance, measurements must be supplemented by validated data and methods of interaction analysis to explain and predict radiation patterns. The National Nuclear Data Center (NNDC) at Brookhaven National Laboratory is active in the development of high energy data bases for radiation transport applications.

A nuclear data file is useful in two ways. Cross-section data are stored and accessed as needed instead of being calculated on demand by lengthy deterministic or Monte Carlo nuclear model calculations. This can not only save time, but also yield unique cross-section values compared to Monte Carlo cross-section calculation methods. The file of reference cross-sections can also be used for sensitivity studies where the effect on calculated results due to discrete changes in input data are observed. This aids cost-benefit analysis since only those data that significantly effect applications need to be seriously reviewed and improved where necessary.

Some features of a nuclear data file are illustrated for high energy protons incident on an Fe-56 target. The nonelastic crosssection for protons on Fe-56 is shown in Figure 1 together with the cross-section for producing secondary neutrons. Empirical fits to the nonelastic cross-section on targets having atomic mass $A$ are similar, varying as $A^{2 / 3}$, $i . e .$, proportional to the nuclear radius squared. The neutron production cross-section increases with energy as the energy thresholds for releasing neutrons are exceeded. Although the sum of the $\sigma(p, n), \sigma(p, p n), \sigma(p, 2 n)$, $\sigma(p, 3 n)$, etc., cross-sections cannot exceed the nonelastic crosssection, the inclusive cross-section for producing neutrons, 


\section{DISCLAIMER}

This report was prepared as an account of work sponsored by an agency of the United States Government. Neither the United States Government nor any agency thereof, nor any of their employees, makes any warranty, express or implied, or assumes any legal liability or responsibility for the accuracy, completeness, or usefulness of any information, apparatus, product, or process disclosed, or represents that its use would not infringe privately owned rights. Reference herein to any specific commercial product, process, or service by trade name, trademark, manufacturer, or otherwise does not necessarily constitute or imply its endorsement, recommendation, or favoring by the United States Government or any agency thereof. The views and opinions of authors expressed herein do not necessarily state or reflect those of the United States Government or any agency thereof. 


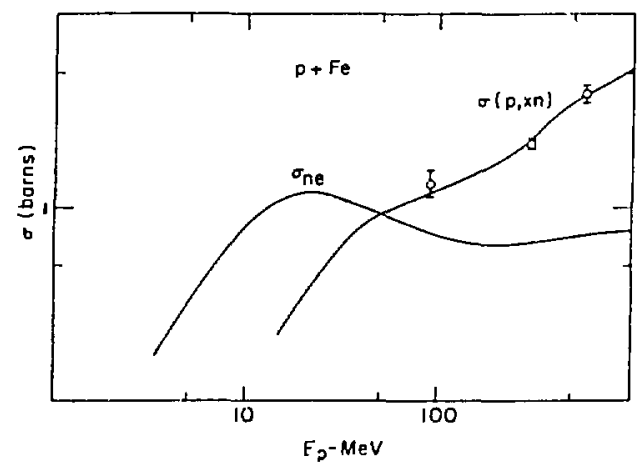

Fig. 1. The iron proton induced nonelastic and ( $p, x n)$ crosssections. The nonelastic cross-section is empirically determined by Letaw et all. The $(p, x n)$ curve is calculated using an extended version of the ALICE code. The experimental $(p, x n)$ cross-sections are inferred from $90 \mathrm{MeV}$ neutron and $160 \mathrm{MeV}$ proton data ${ }^{4}$ and 590 MeV proton data ${ }^{5}$.

$\sigma(p, x n)$, can, since it contains neutron multiplication factors and is equal to the sum of the $\sigma(p, n), \sigma(p, p n), 2 \sigma(p, 2 n), 3 \sigma(p, 3 n)$, etc., quantities. The solid curve for the $\sigma(p, x n)$ cross-section was generated using an extended version ${ }^{2}$ of the ALICE code ${ }^{3}$.

The properties of exclusive reactions such as the $o(p, n)$ cross-section are also important since they determine the production of specific isotopes. A comparison of extended ALICE calculations with experiment ${ }^{6}$ for the Fe-56 $(p, n)$ cross-section is shown in Figure 2. Cross-sections for $\mathrm{Co}_{\mathrm{O}}$ isotopes produced by proton bombardment of $\mathrm{Fe}-56$ were calculated. For isotopes with decreasing numbers of neutrons, the peak cross-section decreases while the cross-section energy threshold increases, as expected. In comparing the calculated isobaric cross-sections for atomic mass $A=54$, the cross-section energy threshold is approximately the same but the peak cross-section for atomic number $z=25$ is highest in the range $z=24$ to 27 . The calculated cross-sections are in general agreement with measurements.

The intranuclear cascade and evaporation nuclear model is generally used to calculate the spallation products of high energy proton reactions. Measured high energy spallation neutrons from the reaction of $590 \mathrm{MeV}$ protogs with $\mathrm{Fe}$ were compared with intranuclear cascade calculations ${ }^{5}$. The high energy neutrons in the forward and backward directions are underestimated by calculations.

The experimental dat $a^{5}$ can be accurately fit at each angle by simple 8 parameter formulas ${ }^{7}$ as shown in Figure 3. The usefulness of the systematics is determined by how well the parametrization can describe measured data at other proton beam energies that were not used in the fitting. Figures 4 and 5 show the results of using systematics determined from $590 \mathrm{MeV}$ proton data to compare with 


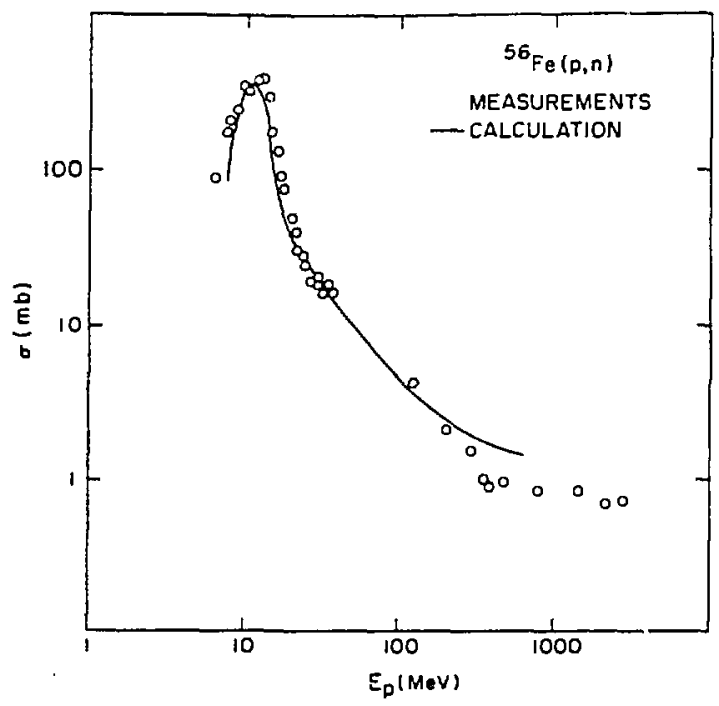

Fig. 2. The iron $(p, n)$ cross-section as a function of incident proton energy. The solid curve is the result of calculations using an extended version of the ALICE code.

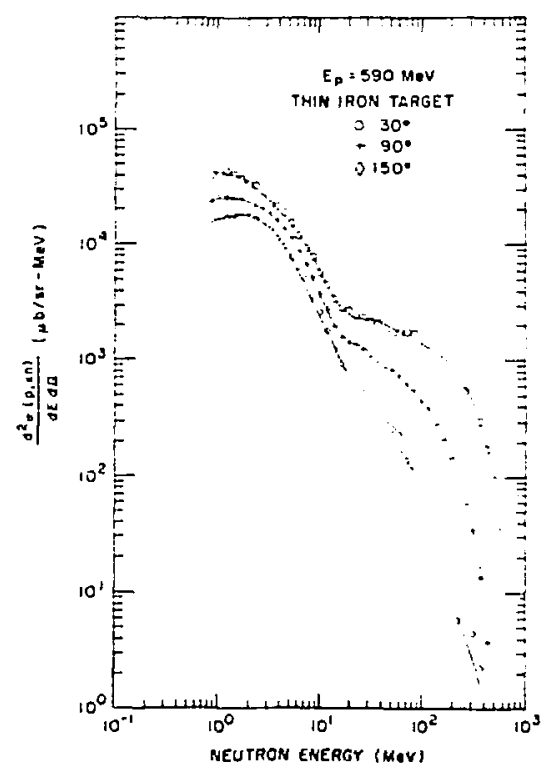

Fig. 3. Double differential neutron emission cross-sections from a thin iron target bombarded by $590 \mathrm{MeV}$ protons. The solid curves are empirical fits to the experimental data. 


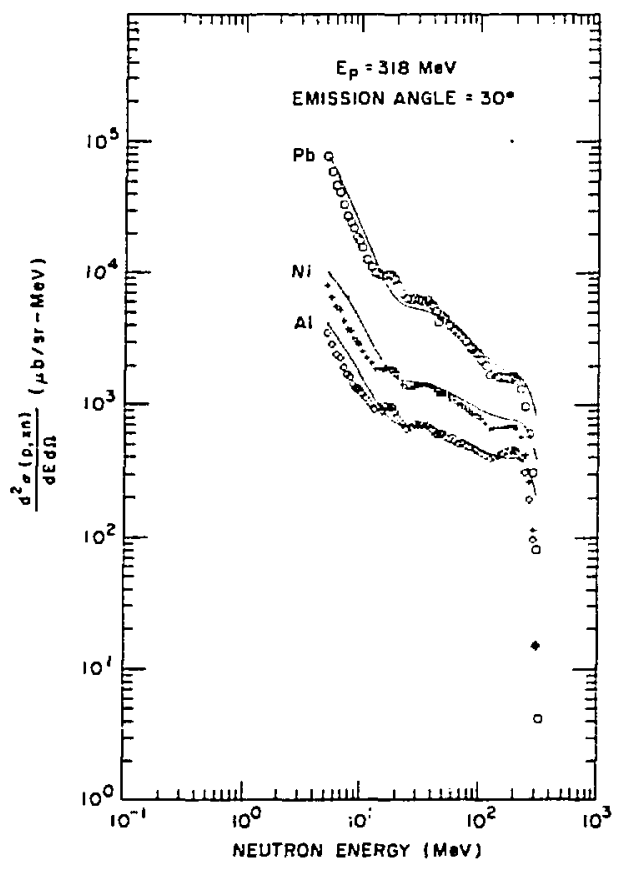

Fig 4. Double differential neutron emission cross-sections at $30^{\circ}$ from thin lead, nickel and aluminum targets bombarded by $318 \mathrm{MeV}$ protons. The solid curves are derived from nuclear systematics.

measurements at 318 and $800 \mathrm{MeV}$, respectively. The energy and angular distributions of high energy neutrons calculated from systematics are significantly closer to experiment than those calculated by the intranuclear cascade nuclear models. The empirically determined parameters may be used to define the crosssection continuously in energy and space as required for radiation transport calculations. The double differential $(p, x n)$ crosssection for $300 \mathrm{MeV}$ protons on $\mathrm{Fe}-56$ is shown in Figure 6 at several neutron emission angles.

The interaction of the neutron fields generated by proton induced spallation reactions with materials in space require the knowledge of high energy neutron cross-sections. The neutron total cross-section has been measured to several GeV for several atomic mass targets. The measured data are stored in NNDC files. A simple parametrization ${ }^{2}$ of the neutron cross-section can describe the data over the atomic mass range equal to or greater than $A=6$ and neutron energies above $1 \mathrm{MeV}$ as shown in Figure 7.

Producing a nuclear data file can include several steps. A search of the bibliography to experimental data is useful to determine what quantitative information is available. A centralized tabulation of experinental data from different 


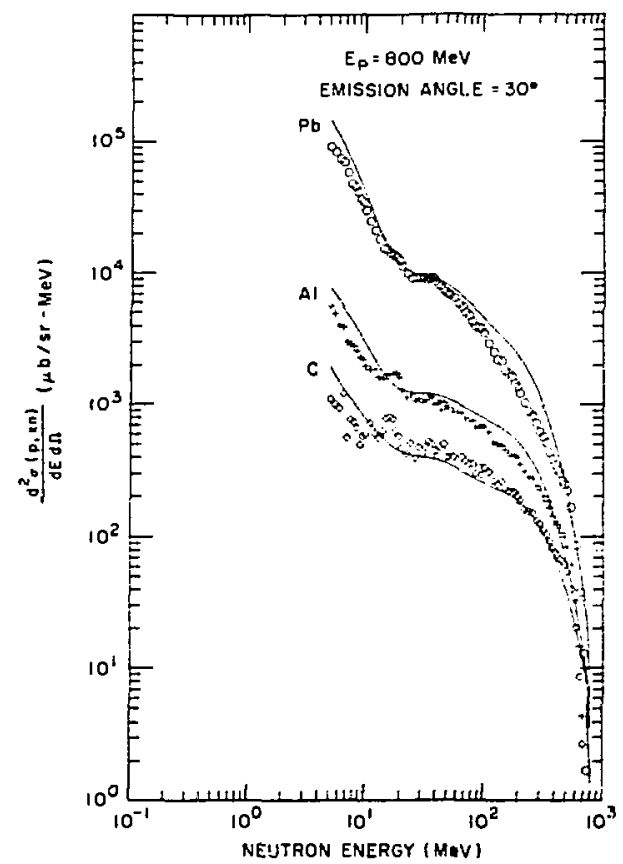

Fig. 5. Double differential neutron emission cross-sections at $30^{\circ}$ from thin lead, aluminum and carbon targets bombarded by $800 \mathrm{MeV}$ protons. The solid curves are derived from nuclear systematics.

measurers, and especially if computerized, can facilitate an intercomparison of measurements and the calculation of additional parameters of interest, e.g., mean energies, integrals, etc. Evaluators must recommend values to use in applications even if experimental results differ, with gaps in measurements filled in by nuclear model calculations or systematics derived from data patterns. The evaluated data should be formatted for convenient use in transport calculations. The data file must be validated for use in applications through calculations of benchmark experiments. Documentation describing the physics content and the computer formatting of the data file ensures correct use of the data and provides reference material when reporting results.

Three indexes to the bibliography are maintained by the NNDC: 1) Neutron Data (CINDA) - bibliographic references to measurements, calculations, reviews and evaluations of microscopic neutron data; 2) Charged Particle Data (CPBIB) - bibliographic references for integral charged-particle reaction data for energies up to $100 \mathrm{MeV}$; and 3) Nuclear Structure and Radioactive Decay Data (NSR) - reference-oriented bihliography of the literature pertaining to nuclear structure, nuclear reactions and radioactive decay data. 


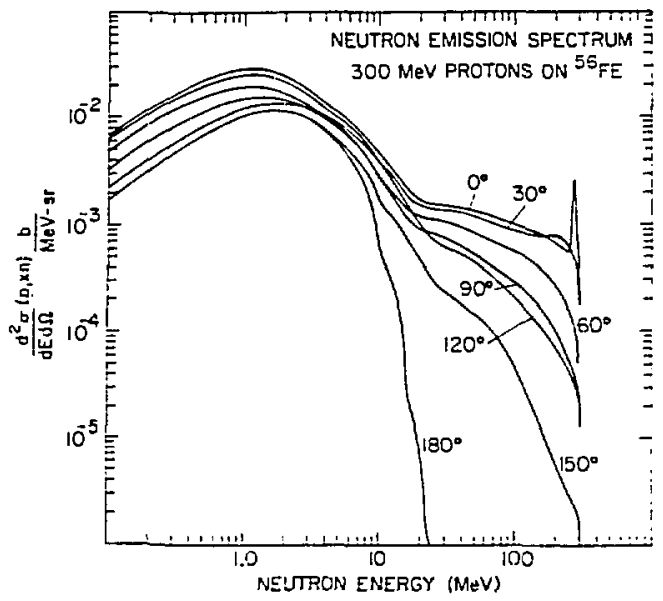

Fig. 6. Evaluated data file contents for the double differential $(p, x n)$ cross-section at several neutron emission angles for $300 \mathrm{MeV}$ protons on $\mathrm{Fe}-56$.

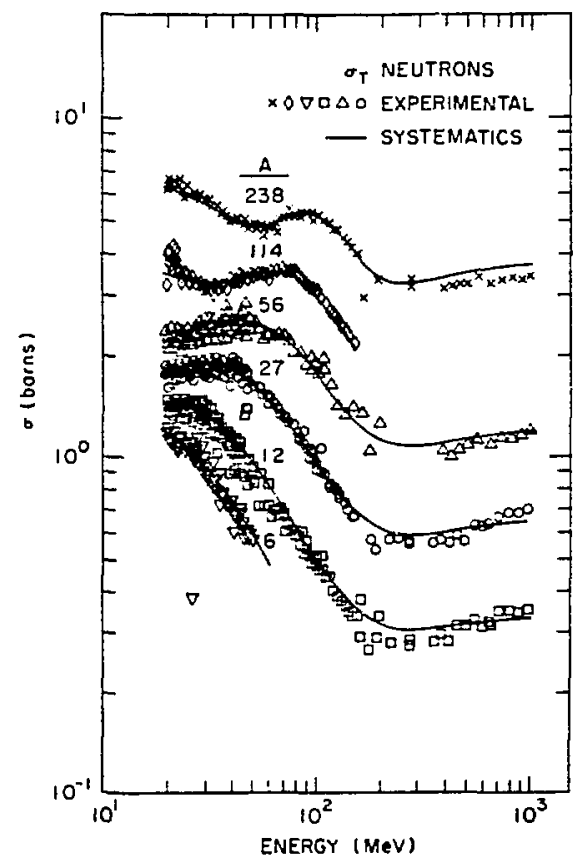

Fig. 7. The neutron induced total cross-section for targets of atomic mass $6,12,27,56,114$, and 238. Experimental data are taken from the NNDC data files. The solid curves are the result of nuclear systematics ${ }^{2}$. 
The CINDA and NSR data bases can be accessed on-line as can other NNDC data bases containing experimental and evaluated data. An atlas of experimental neutron data up to $200 \mathrm{MeV}$ with curves has recently been completed. The complete NNDC services and publications will not be described here, but information can be obtained by contacting the National Nuclear Nata Center, Building 1970, Brookhaven National Laboratory, Upton, NY 11973 or by calling 516-282-2902, (FTS) 666-2901.

\section{RE FERENCES}

1. 1. R. Letaw, C. H. Tsao, and R. Silberberg, Astrophys. J. Supp 1. Ser. 51, 271 (1983).

2. S. Pearlsicein, private communication.

3. M. Blann et al., Phys. Rev. C28, 1648 (1983).

4. W. E. Crandall and G. P. Miliburn, J. App. Phys. 29, 698 (1958).

5. D. Filges et al., Validation of the Intra-Nuclear Cascade Evaporation Model for Particle Production, Jul-1960, November 1984.

6. R. Silberberg and C. H. Tsao, Cross-Sections of Proton-Nucleus Interactions at High Energies, NRL Report 7593, December 1973.

7. S. Pearistein, Nuci. Sci. Eng., 95, 116 (1987).

RESEARCH CARRIED OUT UNDER THE AUSPICES OF THE U.S. DEPARTMENT OF ENERGY UNDER CONTRACT NO. DE-ACO2-76CH00016. 